\title{
Neutral beam heating on the TCV tokamak
}

\author{
Alexander N. Karpushov a,*, René Chavan ${ }^{a}$, Stefano Coda ${ }^{a}$, Vladimir I. Davydenko ${ }^{\mathrm{b}}$, \\ Frédéric Dolizy a ${ }^{a}$ Aleksandr N. Dranitchnikov ${ }^{b}$, Basil P. Duval ${ }^{\mathrm{a}}$, Alexander A. Ivanov ${ }^{\mathrm{b}}$, \\ Damien Fasel $^{\mathrm{a}}$, Ambrogio Fasoli ${ }^{\mathrm{a}}$, Vyacheslav V. Kolmogorov ${ }^{\mathrm{b}}$, Pierre Lavanchy ${ }^{\mathrm{a}}$, \\ Xavier Llobet ${ }^{a}$, Blaise Marlétaz ${ }^{a}$, Philippe Marmillod ${ }^{a}$, Yves Martin ${ }^{a}$, Antoine Merle ${ }^{a}$, \\ Albert Perez $^{\mathrm{a}}$, Olivier Sauter ${ }^{\mathrm{a}}$, Ugo Siravo ${ }^{\mathrm{a}}$, Igor V. Shikhovtsev ${ }^{\mathrm{b}}$, Aleksey V. Sorokin ${ }^{\mathrm{b}}$, \\ Matthieu Toussaint ${ }^{\mathrm{a}}$
}

a Ecole Polytechnique Fédérale de Lausanne (EPFL), Swiss Plasma Center(SPC), CH-1015, Lausanne, Switzerland

${ }^{\mathrm{b}}$ Budker Institute of Nuclear Physics SB RAS, 630090, Novosibirsk, Russia

\section{A R T I C L E I N F O}

\section{Article history:}

Received 13 September 2016

Received in revised form 24 January 2017

Accepted 20 February 2017

Available online xxx

\section{Keywords:}

TCV tokamak

Neutral beam heating

Upgrades

\begin{abstract}
A B S T R A C T
The TCV tokamak contributes to physics understanding in fusion reactor research by harnessing a wide experimental tool set: in particular flexible shaping and high power electron cyclotron heating. Plasma regimes with high plasma pressure, a wider range of temperature ratios and significant fast-ion population are now attainable with a TCV heating system upgrade. In a first stage, a $1 \mathrm{MW}$ neutral beam was installed (2015) and is reported in this paper.

The installation of the NB injector required modifications of the vacuum vessel and considerable work on the machine infrastructure, resulting in a shutdown from late 2013 to mid-2015. TCV is now operating partly as a European Medium-Size Tokamak (MST) facility under the auspices of the EUROfusion consortium. The NBI was intensively operated in the February-July 2016 phase of the MST campaign. Record ion temperatures of $2.0-2.5 \mathrm{keV}$ and toroidal rotation velocities up to $160 \mathrm{~km} / \mathrm{s}$ were promptly attained in the first few L-mode discharges with NB injection. Ion temperatures up to $3.5 \mathrm{keV}$ were subsequently achieved in ELMy H-mode. The injector produces a focused deuterium neutral beam with $25 \mathrm{keV}$ energy, $1 \mathrm{MW}$ neutral power and $2 \mathrm{~s}$ duration.

Highlights:
\end{abstract}

- Installation of $1 \mathrm{MW}, 25 \mathrm{keV}$ neutral beam, direct ion heating, access to Ti/Te $\geq 1$.

- Specific low divergence neutral beam injector with tunable power and energy.

- Ion temperature of $2.0 \mathrm{keV}$, toroidal rotation of $160 \mathrm{~km} / \mathrm{s}$ attained with NB heating.

\section{Introduction}

The Tokamak à Configuration Variable $\left(T C V, R_{O} \cong 0.88 \mathrm{~m}\right.$, $\mathrm{a} \leq 0.25 \mathrm{~m}, \mathrm{~B}_{\mathrm{T}} \leq 1.54 \mathrm{~T}$ ) [1] is characterised by the most extreme plasma shaping capability worldwide (plasma elongation $\kappa$ up to 2.8 , positive and negative triangularity $-0.7 \leq \delta \leq 1$ ), the highest microwave Electron Cyclotron (EC) power density in plasma, and a high degree of flexibility in its heating and control schemes. Main

\footnotetext{
* Corresponding author.

E-mail address: alexander.karpushov@epfl.ch (A.N. Karpushov).
}

TCV missions [2] are to contribute to the physics basis for more efficient ITER exploitation, and optimisation of the tokamak concept, plasma scenarios, heating and control techniques for DEMO and beyond. This requires access to plasma regimes and configurations with high normalised plasma pressure and a wide range of electron/ion temperature ratios, covering $\mathrm{T}_{\mathrm{e}} / \mathrm{T}_{\mathrm{i}} \sim 1$. Implementation of preferential ion heating at the MW power level allows the extension of $T_{i} / T_{e}$ to beyond unity and fills the gap between predominantly electron heated experiments and fusion reactor conditions.

A phased upgrade program [3] is underway on TCV, mainly consisting of adding ion heating (NB injectors), increasing the available electron heating power (X2 and X3 gyrotrons) and installing a 
Table 1

NBI characteristics.

\begin{tabular}{ll}
\hline NB injector reference scenario: & \\
\hline NB power injected in TCV & $1 \mathrm{MW}$ \\
Nominal beam energy & $25 \mathrm{keV}$ \\
Max. NB pulse duration & $2 \mathrm{~s}$ \\
Beam full energy fraction in power & $\geq 70 \%$ \\
NB operation domain: & \\
Beam power range & $0.25 \ldots 1.05 \mathrm{MW}$ \\
Beam energy range & $15 \ldots .25 \mathrm{keV}$ \\
Beam main species & $\mathrm{D}^{\circ} \& \mathrm{H}^{\circ}$ \\
Power sweep response $(\mathrm{P} /(\mathrm{dP} / \mathrm{dt}))$ & $\leq 5 \mathrm{~ms}$ \\
Full power modulation on-time & $5 \mathrm{~ms} . .2 \mathrm{~s}$ \\
Minimal modulation off-time & $5 \mathrm{~ms}$ \\
Modulation rise/fall time & $\leq 0.5 \mathrm{~ms}$ \\
100\% power modulation & up to $200 \mathrm{~Hz}$ \\
\hline
\end{tabular}

divertor structure with variable closure, equipped with gas valves, pumping units and magnetic field coils. A neutral beam injector (NBI), delivering $1 \mathrm{MW}$ power along a tangential (double-pass) line of sight, at energies in the $15-25 \mathrm{keV}$ range, was installed and commissioned, and provided research results in 2016. Two $750 \mathrm{~kW}$, gyrotrons were also commissioned and integrated with three remaining first-generation $500 \mathrm{~kW}$ gyrotrons, providing a projected total of $3 \mathrm{MWX} 2 \mathrm{ECRH}$ power. Two additional $1 \mathrm{MW}$ dualfrequency (X2 and X3) gyrotrons and a second, $1 \mathrm{MW}, 50-60 \mathrm{keV}$ neutral beam are planned.

The ASTRA code was used to simulate the plasma response to combined neutral beam and EC heating in TCV geometry [4]. With the upgraded $(1.5 \rightarrow 3.5 \mathrm{MW}) \mathrm{X} 3 \mathrm{EC}$ system, NBH $(1-2 \mathrm{MW}) \mathrm{TCV}$ could bring the plasma close to the $\beta$-limit in $\mathrm{H}$-mode $\left(\beta_{\mathrm{N}} \sim 2.8\right.$, an important regime for ITER and DEMO), provide direct momentum input to the plasma, and generate a high fast ion fraction for studying wave-particle interaction phenomena of interest for burning plasmas. The $\mathrm{T}_{\mathrm{e}} / \mathrm{T}_{\mathrm{i}} \sim 1$ condition is already met with $\sim 1 \mathrm{MW}$ of NB power with $1 \mathrm{MW}$ of $\mathrm{X} 3 \mathrm{ECH}$. The $\mathrm{T}_{\mathrm{e}} / \mathrm{T}_{\mathrm{i}}$ is expected to vary between 0.5 and 3.0 in TCV's high density (H-mode) confinement regimes.

\section{Neutral beam injector}

TCV's NBI installation was based on considerations of beam access, shine through and orbit losses [5]. A specific geometric arrangement of the NB injection with the beam line at mid plane oriented tangentially relative to the plasma axis was chosen to maximise heating efficiency whilst satisfying machine access limits.

The basic characteristics of TCV's NB system [5] are listed in Table 1 . The $15-25 \mathrm{keV}$ beam energy is safe with respect to orbit losses for $I_{P} \geq 250 \mathrm{kA}$. The $0.25 \ldots 1.05 \mathrm{MW}$ power, tuneable during TCV discharges, enables studies of the plasma reaction to NBH power variation.

The neutral beam injector design is based on a development of the NBI for plasma heating at Budker INP [6]. The injector incorporates a standard positive ion source and elements shown in Fig. 1. An average nominal current density of $0.3 \mathrm{~A} / \mathrm{cm}^{2}$ was chosen for the ion source [5].

The plasma emitter is formed with up to $40 \mathrm{~kW}$ of inductively coupled RF power at $\sim 4 \mathrm{MHz}$ in the plasma box [6] (ceramic aluminium oxide chamber, $346 \mathrm{~mm}$ inner diameter $120 \mathrm{~mm}$ long, Fig. 2-A). A species mix with full, half and $1 / 3$ of acceleration energy of $76: 17: 7 \%$ (power ratios) was measured during the beam commissioning (see Fig. 3).

A high power focused neutral beam with small divergence was developed for the TCV device featuring narrow access ports where only small size, high power density beams can pass. The ballistic beam focusing is provided by spherically shaped multi-aperture electrodes in the ion optical system [7]. Slit apertures in the ion opti-

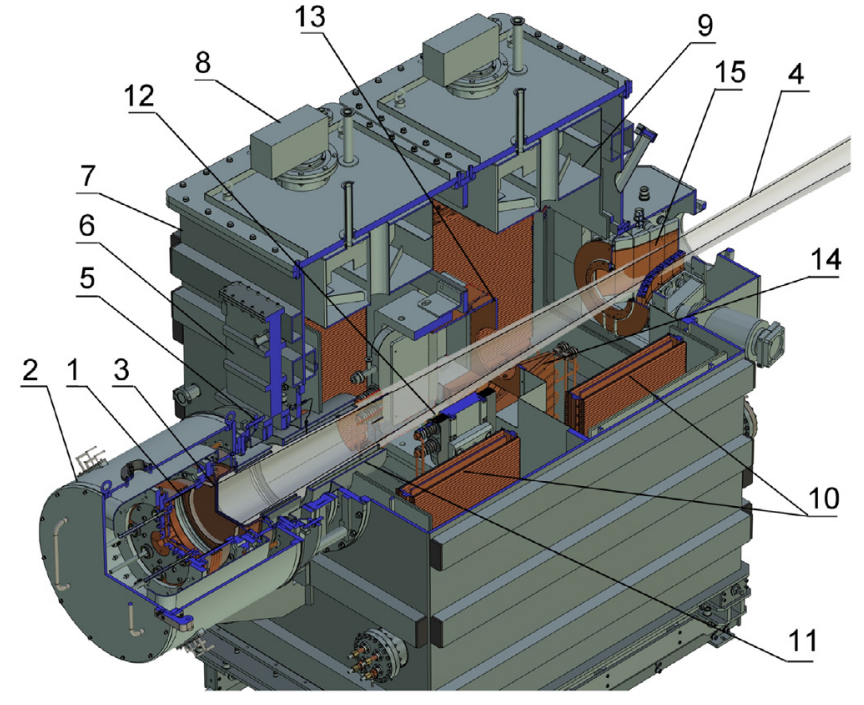

Fig. 1. Neutral beam injector: 1-RF plasma source, 2-magnetic screen, 3-ionoptical system, 4-neutral beam; 5-adjusting device; 6-ions source gate-valve; 7-vacuum tank; 8-cryopump cold head; 9-liquid nitrogen volume; 10-cryo-panels, 11-neutralizer, 12-bending magnet, 13-diaphragm, 14-ion dump for positive ions, 15-calorimeter.
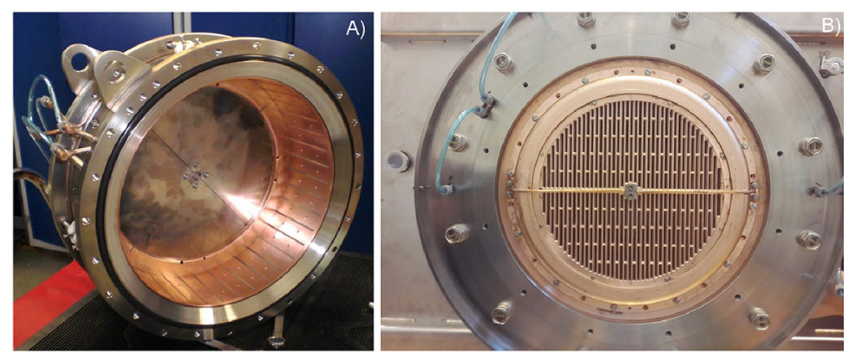

Fig. 2. NBI-TCV plasma box (A) and plasma grid (B).

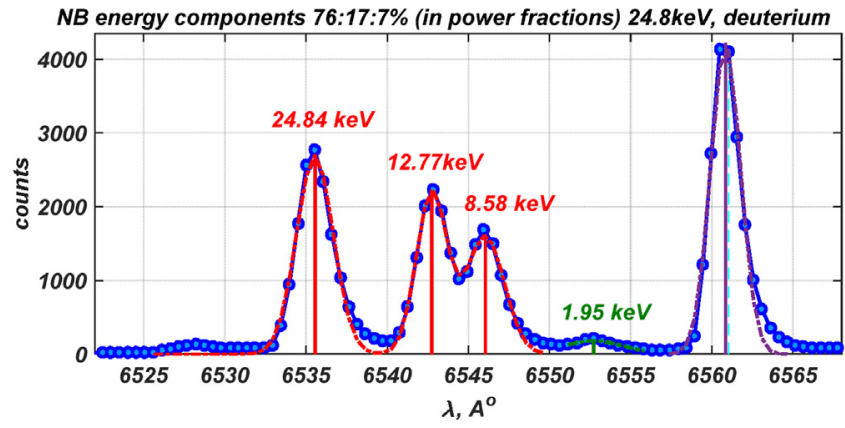

Fig. 3. Neutral beam energy components in power fractions.

cal system reduce the focused beam width in the direction along the slits which is determined by the ion temperature of plasma emitter. $47 \mathrm{~mm}$ long slits with a step of $6 \mathrm{~mm}$ perpendicular to the slits are placed inside the $250 \mathrm{~mm}$ diameter area (Fig. 2-B). The total emission surface of the plasma grid is $224 \mathrm{~cm}^{2}$, corresponding to a transparency of $46 \%$.

The ion source is connected to the vacuum tank through a DN 400 gate valve and a $700 \mathrm{~mm}$ length neutraliser. Two cryo-pumps with total pumping speed of $3 \times 10^{5} \mathrm{l} / \mathrm{s}$ in molecular flow regime for deuterium gas, are used during beam formation. Each cryopump consists of $1.6 \mathrm{~m}^{2}$ surface copper cryopanel cooled by two coldheads (cooling capacity $2 \times 1.5 \mathrm{~W}$ at $4.2 \mathrm{~K}$ ) and a chevron $0.83 \mathrm{~m}^{2}$ 


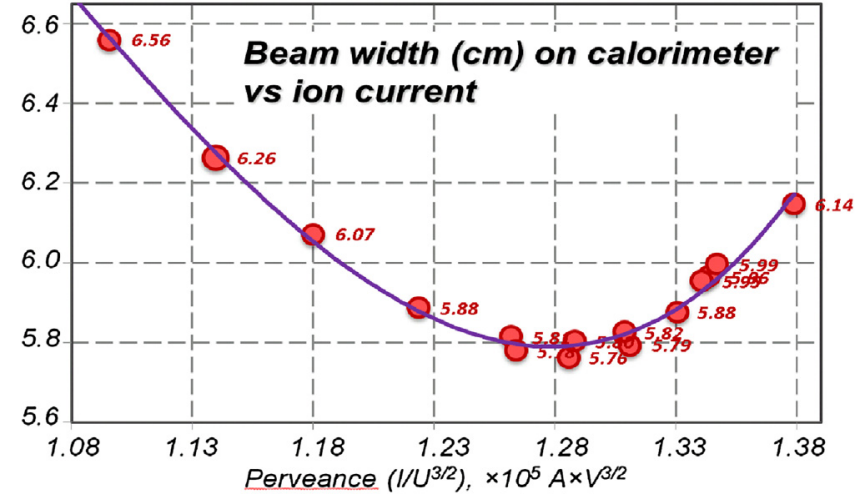

Fig. 4. Example of the perveance scan at $24.8 \mathrm{keV}$.

radiation shield with a transparency of $25 \%$, cooled with liquid nitrogen.

Detectors for the beam alignment (aiming device) and the movable calorimeter are located at the exit of the vacuum tank. A retractable calorimeter can absorb the full duration $(2 \mathrm{~s})$ beam pulse at full power ( $1 \mathrm{MW})$.

Neutral beam operation is overseen by an instrumental computer (LCS) and electronic control modules integrated into the TCV plant control system. This system can handle a large variety of low-voltage analog and digital input/output signals. Protection and interlocks are implemented at the hardware level together with several status monitors and controls. All functions necessary for safe NBI operation are included in the LCS that is designed to protect itself from potentially dangerous external situations and commands.

A beam dump protection system is implemented on TCV to protect against overheat of beam facing elements in the area of beam-wall interaction. The combined RT processing beam inhibit signal generated by plasma disruption detector, a plasma density interlock and direct pyrometric measurements of beam dump surface temperatures are available to the NBI control system.

\section{NBI optimisation and power control}

NBI power control through the plasma discharge is a powerful tool in fusion plasma studies as gradual power ramps up/down permit the investigation of power thresholds for particular processes; e.g. transition between low and high confinement. The $100 \% \mathrm{ON}-$ OFF pulse-width modulation is successful on JET [8] as the time taken to slow down NB fast ions in the plasma is relatively long ( $\sim 100 \mathrm{~ms}$ ) compared to the beam ON/OFF time ( $40 \mathrm{~ms}$ ) and the plasma is therefore relatively insensitive to the modulation process. JET employs 16 independently controlled ion sources (PINIs) to provide time averaged power with a resolution smaller than an individual PINI increment (a similar technique is also used on ASDEX Upgrade). In smaller machines, with a small number of beam sources, a faster fast ion slow down and lower plasma confinement time (TCV, MAST), the plasma would respond strongly to beam modulation, so an alternative power modulation approach is required.

As beam divergence is dependent on both beam current and acceleration voltage (through the perveance), ramping the ion
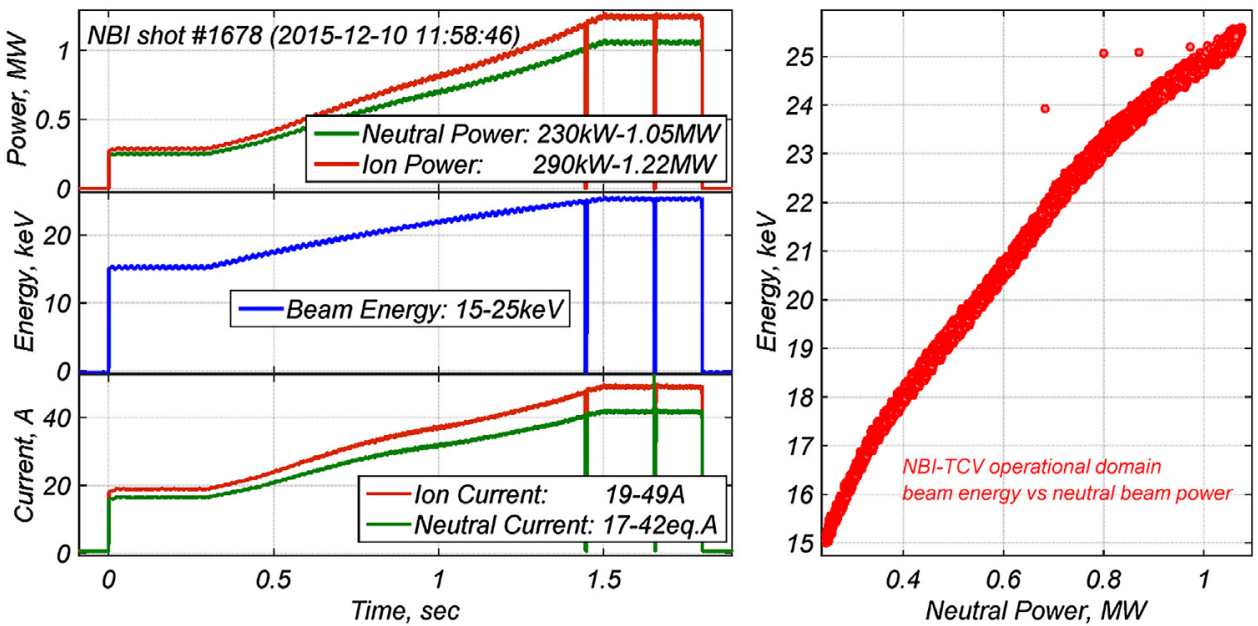

Fig. 5. "Slow" power sweep $(0.23 \ldots 1.05 \mathrm{MW})$ at minimal divergence.
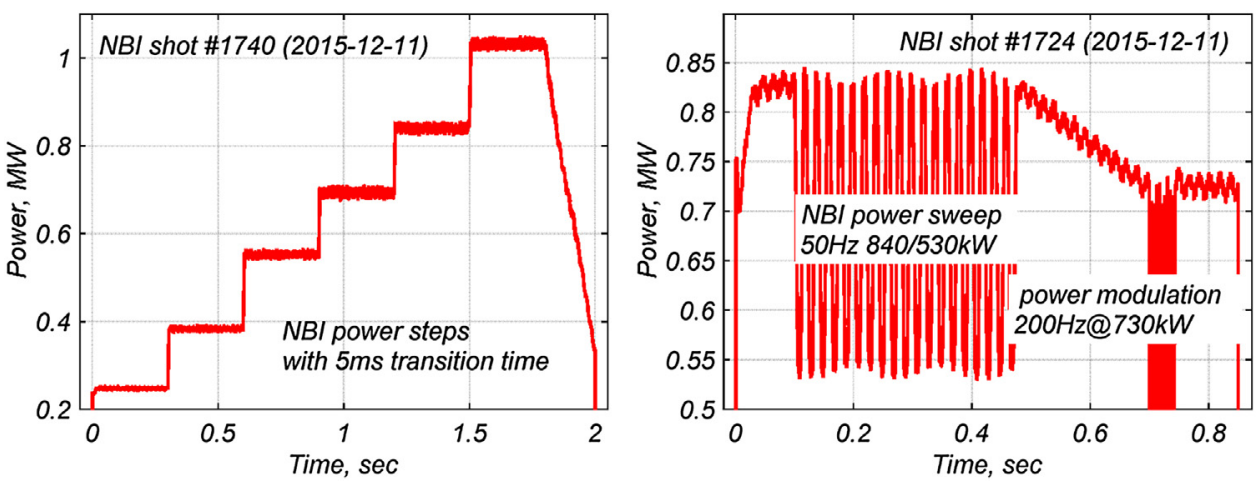

Fig. 6. NBI power steps and modulation during NBI commissioning on the TCV. 
beam current will affect the beam cross section, and beamline transmission. The real time control of an arc current of a high perveance MAST PINI allows variations of the neutral beam power by $\sim 20 \%$ with only minimal effect on the beam footprint [9].

A neutral power variation in the range of $0.25 \ldots 1.0 \mathrm{MW}$ has been implemented on TCV by simultaneous variation of RF power (plasma density in the RF box) and extraction voltage keeping a minimal beam divergence (optimal perveance). The optimisation procedure for the TCV NBI was performed at several (4-6) extraction energies; the optimal beam currents (RF power levels) were experimentally adjusted to minimise the beam divergence; here, minimal divergence (perveance scans) corresponds a minimum of the beam width on the calorimeter (Fig. 4). The voltage on the suppression (2nd) grid and the bending magnet current were also optimised at each power/energy level.

The desired neutral beam power vs time waveform $\left(\mathrm{P}_{0}(t)\right)$ is designed in Matlab. The binary beam ON/OFF, beam energy, neutral and ion currents time traces are calculated accounting for their dependencies on $\mathrm{P}_{0}(\mathrm{t})$ in order to retain a minimal footprint beam width. The digital and analog control waveforms are calculated, transmitted to the LabView LCS program, and uploaded in the FPGA memory of PCIe LCS cards. Following trigger reception, the beam pulse control sequence is executed, and analog and digital control waveforms are transmitted to NBI power supplies. Examples of the TCV-NBI pulses with power variation and modulation are shown in Figs. 5 and 6.

\section{First shots with NB heating on the TCV}

First experiments with NBH (Figs. 7 and 8) demonstrate a core plasma ion temperature in L-mode increasing from about $600-800 \mathrm{eV}$ (typical in TCV at high density in Ohmic plasmas) to $\sim 2 \mathrm{keV}$, in agreement with ASTRA predictions. The plasma rotation with $1 \mathrm{MW}$ NB injection reaches $150-180 \mathrm{~km} / \mathrm{s}$ (CO-NBI direction),

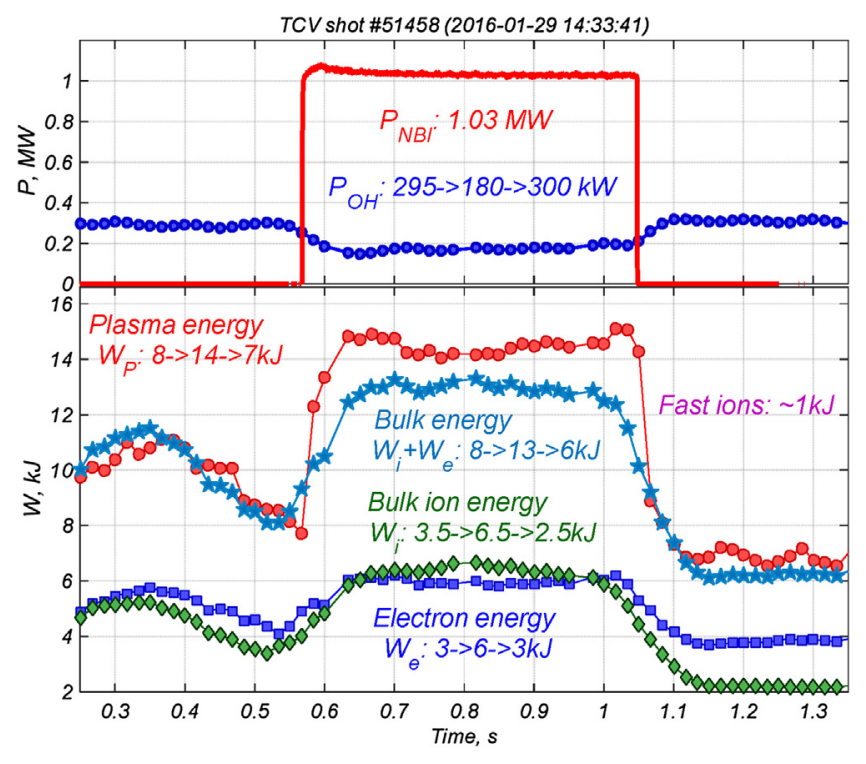

Fig. 7. Global parameters of the TCV plasma with NB heating in L-mode, TCV shot 51458, 5th NBI shot in plasma.

while the typical values for spontaneous intrinsic rotation without NBI are less than $30 \mathrm{~km} / \mathrm{s}$.

The heating neutral beam was intensively used in TCV experiments during the period of February-July 2016, mostly in the MST1 (European Medium-Size Tokamak) experimental program of the EUROfusion consortium. More of $60 \%$ (20 of 33) MST1 experiments used NB heating. $25 \%$ of TCV discharges ( 579 shots) used NB injection into plasma during this period. Beam availability was $85-90 \%$, with most (7-10\%) faults in NB injection in TCV related to problems with NBI control electronics and power supplies. The total energy delivered by the NBI into TCV was limited to $1 / 4(0.5 \mathrm{MJ})$ of the design
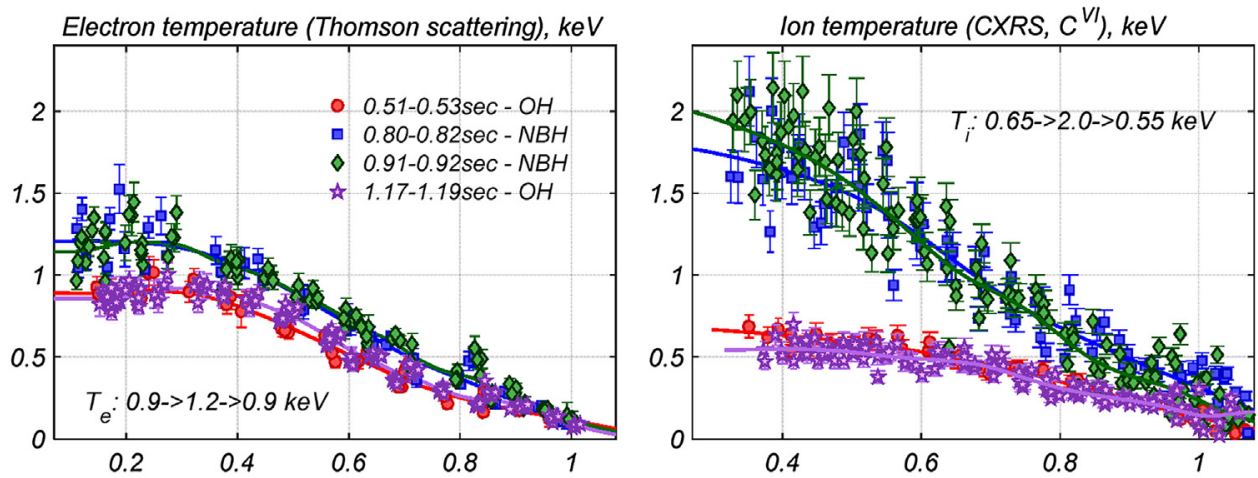

Electron density (Thomson scattering), $10^{19} \mathrm{~m}^{-3}$
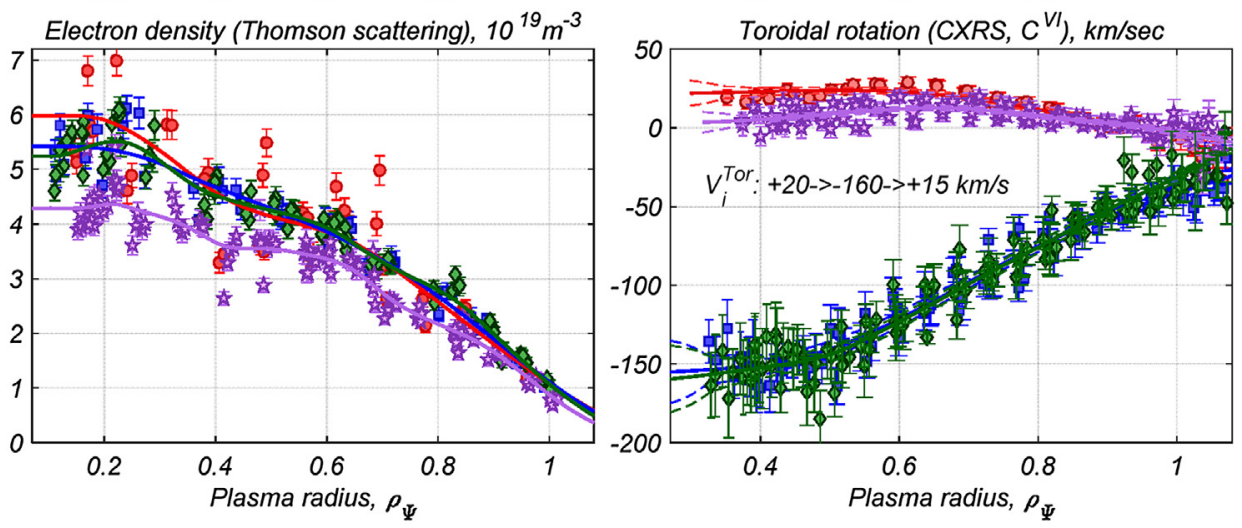

Fig. 8. Plasma radial profiles with and without NBI in L-mode; TCV shot 51458. 

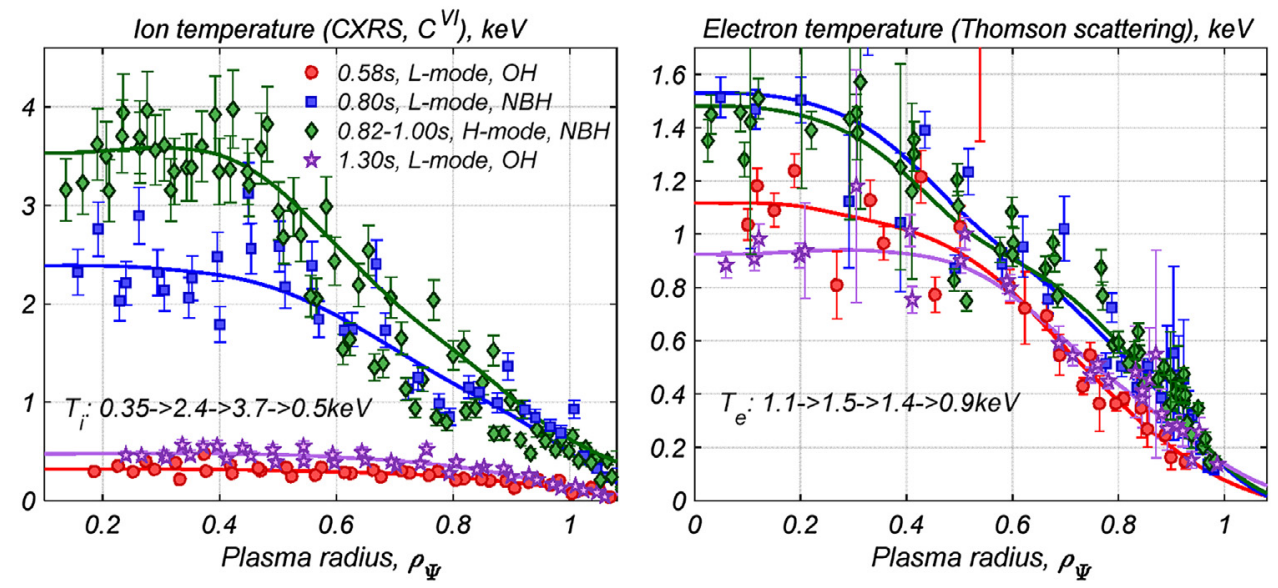

Fig. 9. Ion and electron temperature profiles in the ELMy H-mode discharge, TCV shot \#53362.

value (1 MW, 2 s) due to non-optimal angular characteristics (divergence or/and focal length) of the beam compared to the beam duct, and the subsequent overheating that this provoked. Resolution of this problem is ongoing and will include a modification of the beam duct and improved ion optics (grids).

The TCV record ion temperature of $3.7 \mathrm{keV}$ (Fig. 9) was achieved in the MST1 high confinement ELMy H-mode experiments. NB injection on TCV facilitates $\mathrm{H}$-mode access, changes sawtooth and ELM frequencies, and provides a significant (up to $70 \mathrm{kA}$ ) plasma current drive.

With the installation of the first $1 \mathrm{MW}$ neutral beam TCV has greatly extended the range of accessible plasma parameters that are highly relevant to tokamak fundamental physics and machine operation studies and will strongly contribute to the ITER and DEMO projects.

\section{Acknowledgments}

The authors are very grateful to Manfred Sauer (ex. Inst. fur Plasmaphys., Forschungszentrum Julich $\mathrm{GmbH}$ ) for participation in the NBI installation and commissioning on TCV, to Dr. David L. Keeling and Tim Robinson (EURATOM/CCFE Fusion Association, Culham Science Centre) for useful discussions and help with NBI operation during the EUROfusion MST1 campaign, to members of SPC-EPFL and Budker INP teams involved in the work on manufacturing, installation, commissioning and operation of heating beam.
This work was supported in part by the Swiss National Science Foundation. This work has been carried out within the framework of the EUROfusion Consortium and has received funding from the Euratom research and training programme 2014-2018 under grant agreement No 633053. The views and opinions expressed herein do not necessarily reflect those of the European Commission.

\section{References}

[1] A. Fasoli, for the TCV Team, Overview of physics research on the TCV tokamak, Nucl. Fusion 49 (2009) 104005.

[2] S. Coda, for the TCV Team, The science program of the TCV tokamak: exploring fusion reactor and power plant concepts, Nucl. Fusion 55 (2015) 104004

[3] A. Fasoli, for the TCV Team, TCV heating and in-vessel upgrades for addressing DEMO physics issues, Nucl. Fusion 55 (2015) 043006.

[4] A.N. Karpushov, et al., A scoping study of the application of neutral beam heating on the TCV tokamak, Fusion Eng. Des. 86 (2011) 868.

[5] A.N. Karpushov, et al., Upgrade of the TCV tokamak, first phase: neutral beam heating system, Fusion Eng. Des. 493 (2015) 96-97.

[6] A. Sorokin, et al., Characterization of $1 \mathrm{MW} 40 \mathrm{keV}, 1 \mathrm{~s}$ neutral beam for plasma heating, Rev. Sci. Instrum. 81 (2010) 02B108.

[7] V. Davydenko, et al., Multi-slit triode ion optical system with ballistic beam focusing, Rev. Sci. Instrum. 87 (2016) 02B303.

[8] D. Ćirić, et al., Performance of upgraded JET neutral beam injectors, Fusion Eng. Des. 86 (2011) 509.

[9] D.A. Homfray, et al., Real time neutral beam power control on MAST, Fusion Eng. Des. 86 (2011) 780. 